\title{
Plasmonic nanoparticle enhanced and extended performance of Light-sensitive nanocrystal skins
}

\author{
Shahab Akhavan ${ }^{1}$, Kivanc Gungor ${ }^{1}$, and Hilmi Volkan Demir ${ }^{1,2}$ \\ ${ }^{1}$ UNAM-Institute of Materials Science and Nanotechnology, Department of Electrical and \\ Electronics Engineering, Department of Physics, Bilkent University, Ankara, 06800, Turkey \\ ${ }^{2}$ School of Electrical and Electronic Engineering, School of Physical and Mathematical Sciences, \\ Nanyang Technological University, Singapore, 639798, Singapore
}

\begin{abstract}
We report on light-sensitive nanocrystal skin (LS-NS) platforms composed of monolayer visible nanocrystals (NCs) on top of bilayers of polyelectrolyte polymers. These LS-NS devices are operated on the principle of photogenerated potential buildup, unlike common photodetectors that operate on the basis of charge collection. The resulting devices are as highly sensitive as common photosensors, despite utilizing a monolayer of NCs and requiring no applied external bias. In this device architecture, using only a single $\mathrm{NC}$ monolayer also allows to reduce noise current generation. This LS-NS platform is highly stable under ambient conditions with fully sealed NC monolayer, promising for low-cost large-area UV/visible sensing applications. However, such visible NC based LS-NS devices exhibit limited performance in the long wavelength range due to the low optical absorption of these NCs (e.g., CdTe NCs) in this spectral range. Here, to enhance the device sensitivity, incorporating silver nanoparticles into LS-NS is proposed and demonstrated. For that, the optical absorption of CdTe monolayer NCs in the LS-NS devices is increased using the embedded silver nanostructures. With plasmon coupling, we observe a 2.6-fold enhancement factor in the photosensitivity around the localized surface plasmonic resonance peak of the nanostructures. Higher sensitivity improvement is also obtained at longer wavelengths. To predict the enhancement in the sensitivity of the LS-NS, numerical simulations are performed and the simulation results are found to agree well with the experimental data. Plasmonically enhanced LS-NS hold great promise for large-area photosensing applications extending from UV to IR including windows and facades of smart buildings.
\end{abstract}

\section{INTRODUCTION}

Colloidal semiconductor nanocrystals (NCs) are considered to make strong candidates as an alternative class of materials to replace conventional semiconductor epitaxial materials as well as rare-earth materials in various applications. NCs offer great potential for integrating them into optoelectronic devices because of their superior optical properties including bandgap tunability [1]. Since these materials have narrow emission and broad absorption bands, it is possible to control and fine-tune their emission spectrum while being able to optically excite them conveniently at short wavelengths, e.g., for color-conversion LEDs [2]. These materials are also considered as prospective materials to be used in photovoltaics and light sensing [3-4]. In addition to the aforementioned advantages of NCs, the ability to easily deposit NCs via spray- 
coating, dip-coating and spin-coating at reduced costs over large areas, where the lattice mismatch does not arise as a problem, motivates us to fabricate highly sensitive large-area photosensing devices $\left(48 \mathrm{~cm}^{2}\right)$, which can be fabricated easily also on flexible substrates (see Figure 1). Using this approach, semi-transparent large-area LS-NS platform is demonstrated via spray-coating visible NCs on top of polyelectrolyte-polymers, which is highly sensitive to UV and visible light [5].

These LS-NS devices are operated on the basis of photogenerated potential buildup, as opposed to conventional charge collection. Close interaction of NCs with the top contact, while the other side of the device is isolated using a high dielectric spacing layer, resulted in high sensitivity in the absence of external bias. Furthermore, a single NC monolayer makes the device semi-transparent with sufficient optical absorption, while reducing noise generation and dark current. However, visible NCs used in these LS-NS devices show small absorption at long wavelengths, which limit the device performance. To enhance the sensitivity of the devices, embedding silver nanostructures underneath the NC monolayer is proposed and demonstrated. Using silver nanostructures, we optimize the localized surface plasmonic enhancement by tuning the plasmonic resonance and spacing between the plasmonic nanostructure and semiconductor $\mathrm{NC}$ materials.

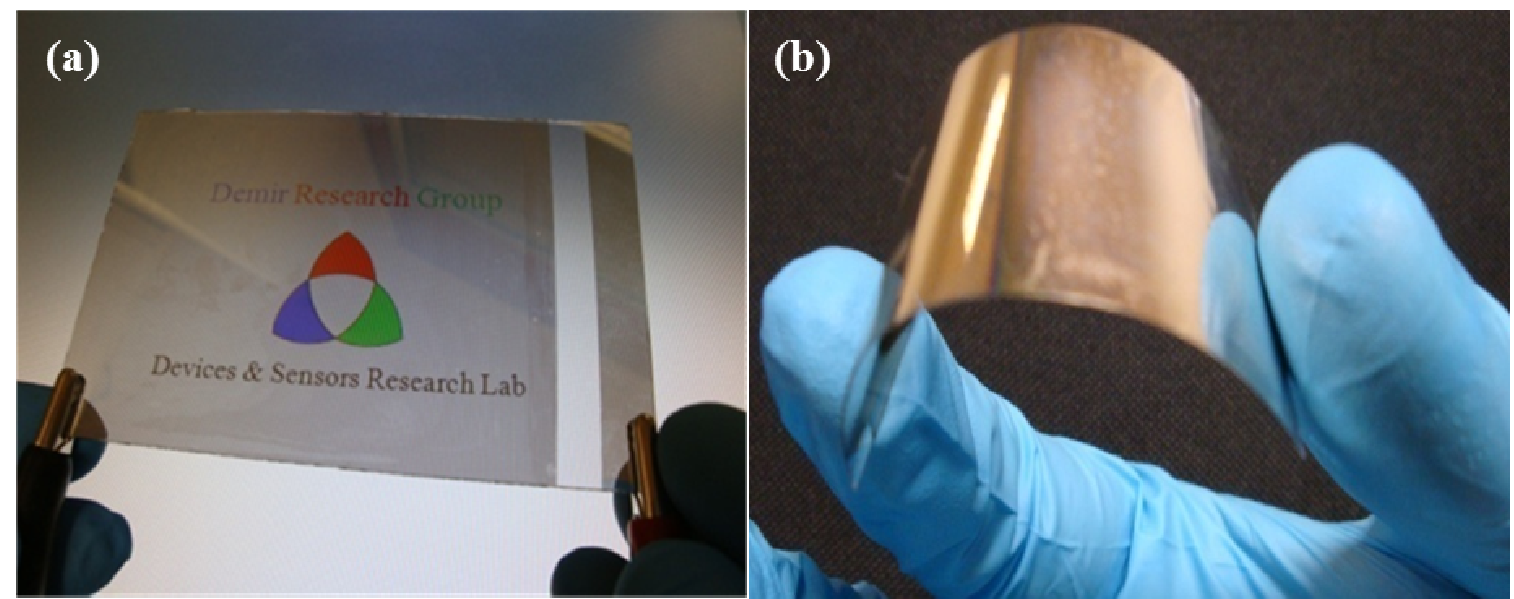

Figure 1. (a) Large area LS-NS ( $8 \mathrm{~cm} \times 6 \mathrm{~cm}$ in dimension) and (b) photograph of a flexible LS-NS composed of a monolayer of TGA capped CdTe NCs on the top of polyelectrolyte polymers (PDDA and PSS) [5].

\section{EXPERIMENTS AND DISCUSSION}

To fabricate the LS-NS device, ITO film on a glass (or PET) substrate is sonicated in a mixture of $2 \mathrm{~mL}$ Hellmanex in $100 \mathrm{~mL}$ water for $15 \mathrm{~min}$, followed by cleaning in Milli-Q water (15 min), acetone $(15 \mathrm{~min})$ and isopropanol $(15 \mathrm{~min})$. Then, we deposit $50 \mathrm{~nm} \mathrm{HfO}_{2}$ dielectric film using atomic layer deposition (ALD). To obtain localized plasmonic nanostructures we lay down silver nanoparticles via thermal evaporator with $10 \mathrm{~nm}$ mass thickness. To reorganize the silver nanoparticles into the desired morphology, we anneal the sample at different temperatures for different durations $\left(150{ }^{\circ} \mathrm{C}\right.$ for $2 \mathrm{~min}, 300{ }^{\circ} \mathrm{C}$ for $2 \mathrm{~min}$, and $300{ }^{\circ} \mathrm{C}$ for $20 \mathrm{~min}$ ) using rapid thermal annealing to obtain the best localized surface plasmonic (LSP) peak. Subsequently, heat treatment helps the silver nanoparticles to reorganize themselves such that they become more isolated from each other and more spherical in shape, as shown in Figure 2(a-d). 

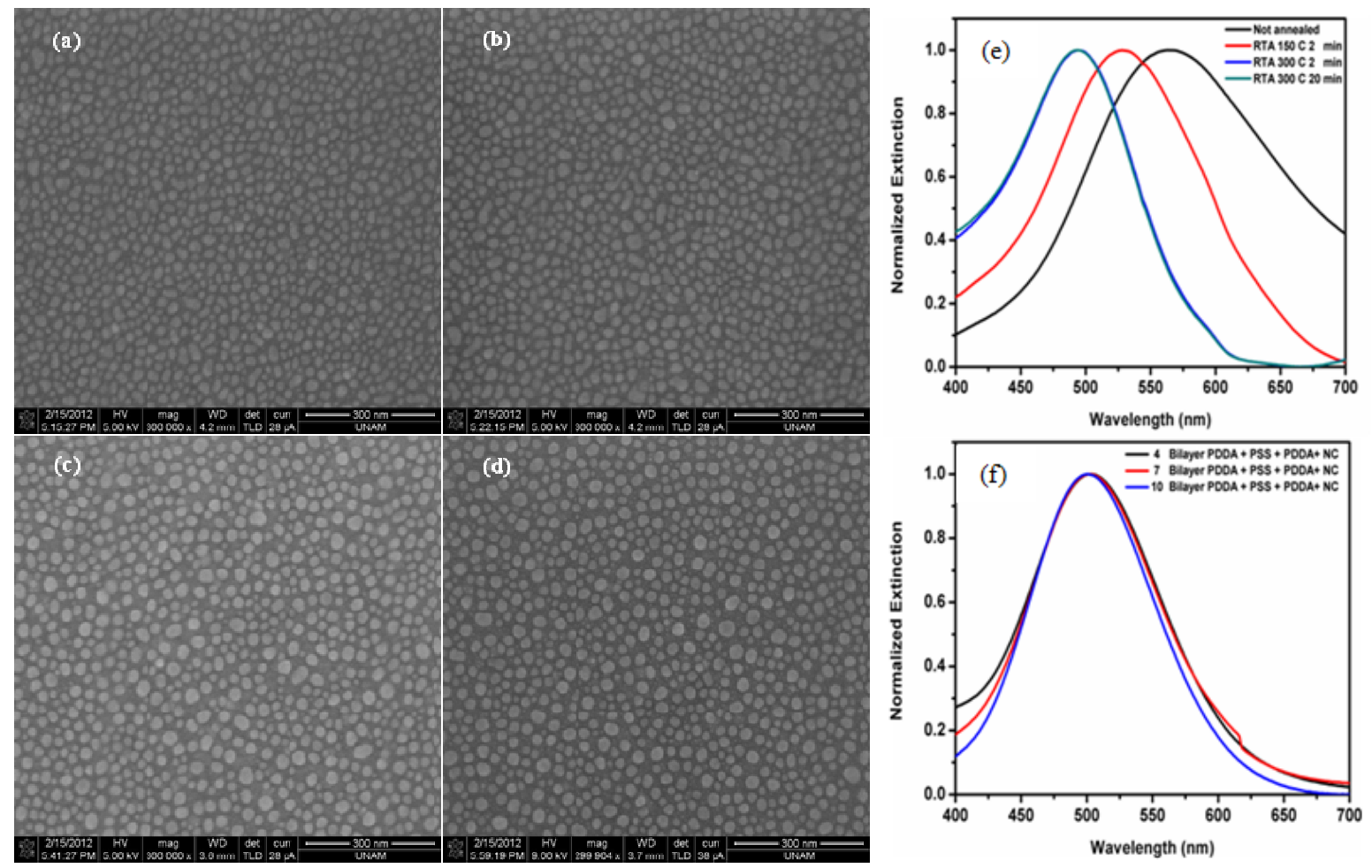

Figure 2. Scanning electron microscopy (SEM) images of four silver nanoparticle films with 10 nm mass thickness; (a) not annealed, (b) annealed at $150{ }^{\circ} \mathrm{C}$ for $2 \mathrm{~min}$, (c) annealed at $300{ }^{\circ} \mathrm{C}$ for $2 \mathrm{~min}$, and (d) annealed at $300{ }^{\circ} \mathrm{C}$ for $20 \mathrm{~min}$. (e) Corresponding optical absorption spectra of silver nanoparticle films. (f) Optical absorption spectra of silver nanoparticle film annealed at $300{ }^{\circ} \mathrm{C}$ for 20 min covered with $1 \mathrm{~nm} \mathrm{Al} \mathrm{O}_{3}$ and various bilayers of PDDA and PSS [6].

As it can be seen from Figures 2(e-f) and 2(a-d), the optical excitation spectra and scanning electron microscopy (SEM) images are in agreement with each other. Isolation and size reduction of nanoparticles result in narrower FWHM, accompanied with a blue-shifted and stronger LSP resonance. To further predict the enhancement in photosensitivity of the LS-NS, we perform numerical simulations using commercially available software FDTD and observe near field electric field enhancement in the vicinity of these silver nanoparticles, as depicted in Figure 3. 

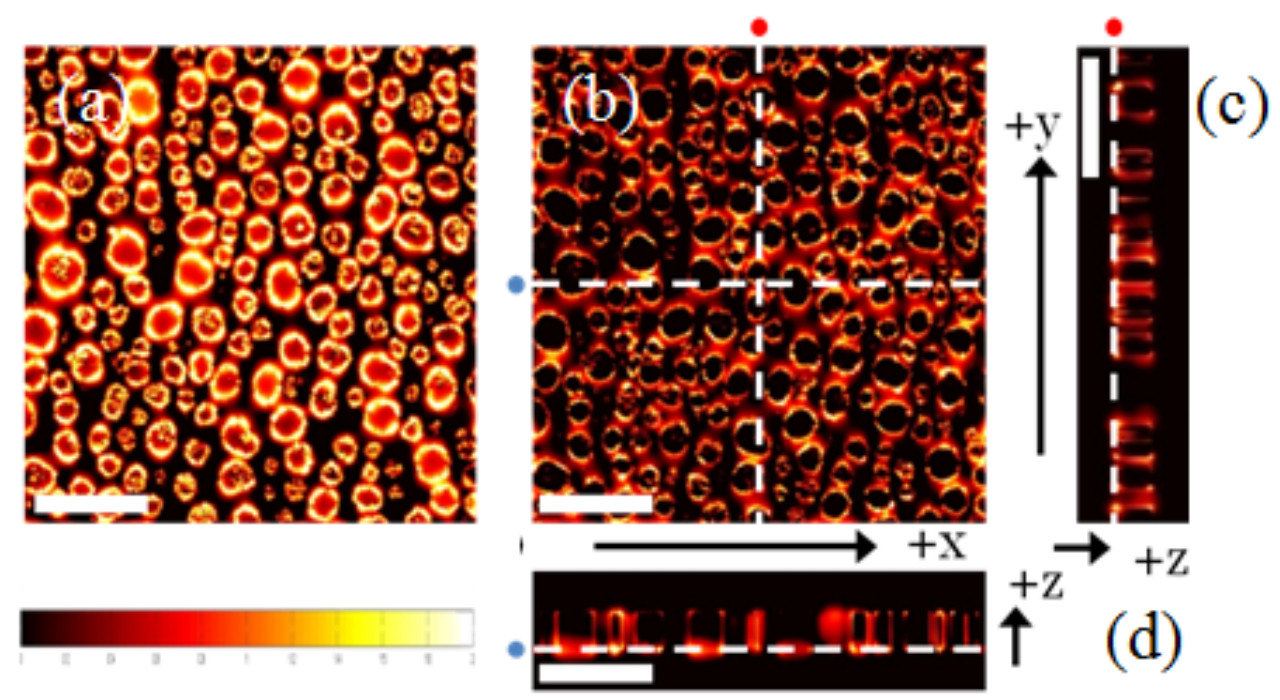

Figure 3. (a) Intensity distribution of electric field at the interface of silver nanoparticles and $\mathrm{HfO}_{2}$ film. (b) Intensity distribution of electric field along $x-y$ plane of the air interface and silver nanoparticles. (c) 2D cross-sectional intensity distribution of electric field along y-z plane. White dashed line indicated with red dots coincides with the vertical line of Figure 3(b). (d) 2D cross-sectional intensity distribution of electric field along X-Z plane. White dashed line indicated with blue dots coincides with the horizontal line of Figure 3(b) [6].

Subsequently, to deposit NCs we use LBL self-assembly via dip coating, which relies on alternating adsorption of positively poly(diallyldimethylammonium chloride) (PDDA) and negatively poly(sodium 4-styrenesulfonate) (PSS) charged polyelectrolyte pairs. LBL films are prepared as follows: (i) dipping silver nanoparticle film into a PDDA solution for $5 \mathrm{~min}$, (ii) cleaning it with water for $1 \mathrm{~min}$, (iii) next dipping it into a solution of PSS for $5 \mathrm{~min}$, and (iv) again washing it with water for 1 min. Finally, after deposition of one extra layer of PDDA and washing with water, follow the steps of dipping the sample into the dispersion of CdTe NCs for $20 \mathrm{~min}$ and rinsing with water again for $1 \mathrm{~min}$. To remove excess and unbound species of the substrate the sample is washed after each self-assembly step. Finally, Al contact layer is deposited immediately on top of the CdTe $\mathrm{NC}$ monolayer via thermal evaporation. To characterize the LS-NS, we use Agilent Technologies B1500A semiconductor parameter analyzer and Xenon light source with an integrated monochromator. The illumination intensity is measured with an optical power meter (Newport multi-function optical power meter). During these measurements, the samples are grounded from the ITO side and connected with a load resistance of $200 \mathrm{M} \Omega$ to both sides of the contact. During operation, no external bias is applied across the device.

Following electron and hole pair generation, charges are not allowed to pass through the device. Due to the device design structure and work function of NCs and top aluminum contact, holes are accumulated at the Al contact. Indeed, in this case, the metal contact acts as the hole acceptor, while the remaining electrons are trapped inside the NCs. We observe that a higher voltage buildup can be obtained at shorter wavelengths due to the higher absorption of CdTe NCs where more states are available for electron and hole pair generation. This is in strong agreement with the CdTe absorption spectrum. In order to enhance the small absorption of NCs in the long wavelength region, which limits the device performance, silver nanostructure are embedded into LS-NS. Tuning metallic silver nanostructures, we optimize the localized 
plasmonic enhancement by shifting the plasmonic resonance and the spacing between the plasmonic structure and semiconductor materials. Various bilayers of polyelectrolyte polymers (PDDA and PSS) deposited on $1 \mathrm{~nm} \mathrm{Al}_{2} \mathrm{O}_{3}$ as the interparticle distance between the metal nanoparticle and NCs, in order to facilitate the effects of distance dependent behavior on localized plasmonic enhancement. Consequently, we find that localized plasmon enhancement is possible for the spacing of about $8-15 \mathrm{~nm}$ in our device. Subsequently, the presence of metal nanoparticles results in enhanced absorption of the CdTe NCs film to generate more electrons and holes. As a result, more voltage buildup can be obtained to enhance the sensitivity of the device. With plasmon coupling, the deposited plasmonic silver nanoparticles increased optical absorption of the NC monolayer and enhanced the device photosensitivity. Improvements in photosensitivity over broadband (400-650 nm) spectral range are observed with a 2.6-fold enhancement factor around the localized surface plasmon resonance peak. Higher sensitivity intensification is also obtained at longer wavelengths and the device operation is extended (see Figure 4).
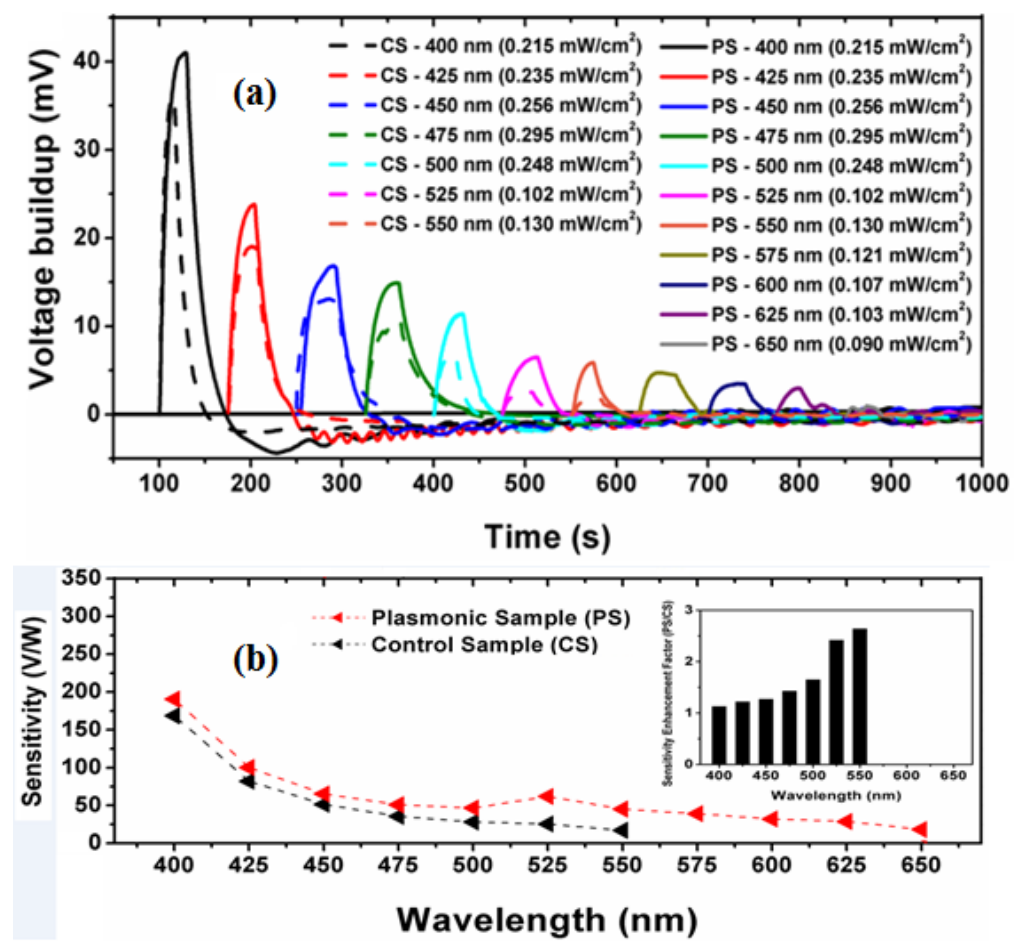

Figure 4. (a) Voltage buildup variations of LS-NS device for different excitation wavelengths. Solid line represents the plasmonic sample (PS) and dashed line represents the control sample (CS). (b) Comparison of the sensitivity of LS-NS device structure in the absence and presence of plasmonic nanostructures. Inset figure presents the sensitivity enhancement factor vs. wavelength.

\section{CONCLUSIONS}

In conclusion, we demonstrated that using the silver nanostructures the photosensitivity of LSNS devices can be enhanced. Furthermore, the operational wavelength region can also be extended. With numerical simulations, we expected considerable increase in the vicinity of silver 
nanoparticles from the electric field intensity distributions, as also observed in the experimentally measured sensitivity of the device. Our findings presented herein have significant implications for the future design of $\mathrm{NC}$ thin film based optical sensing platforms.

\section{ACKNOWLEDGMENTS}

We gratefully acknowledge the financial support in part by ESF EURYI, EU FP7 Nanophotonics4Energy NoE, and TUBITAK under the Project No. EEEAG 110E217 and 111E186, and in part by NRF-CRP6-2010-02 and NRF RF 2009-09. HVD acknowledges additional support from TUBA-GEBIP.

\section{REFERENCES}

1. S. V. Gaponenko, Introduction to Nanophotonics (Cambridge University Press, 2010).

2. H. V. Demir, S. Nizamoglu, T. Erdem, E. Mutlugun, N. Gaponik, and A. Eychmüller, "Quantum dot integrated LEDs using photonic and excitonic color conversion," Nano Today 6(6), 632-647 (2011).

3. J. M. Luther, M. Law, M. C. Beard, Q. Song, M. O. Reese, R. J. Ellingson, and A. J. Nozik, "Schottky solar cells based on colloidal nanocrystal films," Nano Lett. 8(10), 3488-3492 (2008).

4. G. Konstantatos and E. H. Sargent, "Nanostructured materials for photon detection," Nature Nanotech. 5, 391-400 (2010).

5. S. Akhavan, B. Guzelturk, V. K. Sharma, and H. V. Demir, "Large-area semi-transparent light-sensitive nanocrystal skins," Optics Express 20 (23) 25255-25266 (2012).

6. S. Akhavan, et al. (submitted) 